\title{
On the influence of noise statistics on polarimetric contrast optimization
}

\author{
Guillaume Anna, ${ }^{1}$ François Goudail, ${ }^{1, \star}$ Pierre Chavel, ${ }^{1}$ and Daniel Dolfi' \\ 'Laboratoire Charles Fabry de l'Institut d'Optique, CNRS, Univ Paris-Sud, Campus Polytechnique, \\ RD 128, 91127 Palaiseau, France \\ ${ }^{2}$ Thales Research and Technology_France, RD128, 91767 Palaiseau Cedex, France \\ ${ }^{*}$ Corresponding author: francois.goudail@ institutoptique.fr
}

Received 9 September 2011; accepted 12 January 2012;

posted 18 January 2012 (Doc. ID 154446); published 9 March 2012

\begin{abstract}
In active scalar polarimetric imaging systems, the illumination and analysis polarization states are degrees of freedom that can be used to maximize the performance. These optimal states depend on the statistics of the noise that perturbs image acquisition. We investigate the problem of optimization of discrimination ability (contrast) of such imagers in the presence of three different types of noise statistics frequently encountered in optical images (Gaussian, Poisson, and Gamma). To compare these different situations within a common theoretical framework, we use the Bhattacharyya distance and the Fisher ratio as measures of contrast. We show that the optimal states depend on a trade-off between the target/ background intensity difference and the average intensity in the acquired image, and that this trade-off depends on the noise statistics. On a few examples, we show that the gain in contrast obtained by implementing the states adapted to the noise statistics actually present in the image can be significant. (C) 2012 Optical Society of America

OCIS codes: $260.5430,030.4280$.
\end{abstract}

\section{Introduction}

Polarimetric images are obtained by measuring some characteristics of the polarization state of the light scattered by a scene. They can reveal contrasts that do not appear in classical intensity images and have found more and more applications in remote sensing, biomedical imaging, and industrial control [1-3] . The technologies for developing such imagers are now mature and it is, therefore, possible to address their optimization. The criterion for this optimization depends on the information that is expected from the images. In this paper, we shall address the problem of discrimination between two regions of a scene that have different polarimetric properties, by using imaging systems in which the illumination and analysis states are controlled. One important domain of application of these results is target

$1559-128 \mathrm{X} / 12 / 081178-10 \$ 15.00 / 0$

(C) 2012 Optical Society of America detection. This issue has first been addressed in the radar community [4-6]. Because of the specific characteristics of radar systems, speckle noise was implicitly assumed for modeling image variability. More recently, optimization of the contrast in optical polarimetric systems has been addressed [7-9]. In particular, it has been shown that, in the presence of additive noise, the optimal discrimination is reached by performing a single acquisition of an intensity image with optimized illumination and analysis states [10]. Furthermore, optimization in the presence of spatial variations in the scene has been addressed [11].

The purpose of the current paper is to present and compare contrast optimization methods in images perturbed by the three types of noise that are most frequently encountered in optics: additive Gaussian noise, representative of sensor noise, Poisson shot noise that results from the quantum fluctuations of the useful or ambient light flux, and Gamma 
distributed speckle noise, encountered when illuminating the scene with coherent light. To include them in a common framework, we use the Bhattacharyya distance as a measure of the contrast [12], and the Fisher ratio as an easy-to-interpret approximation. This framework will have the further advantage that systems perturbed with different types of noise can be compared. To the best of our knowledge, optimization of the contrast in the presence of dominant Poisson shot noise and comparison of the optimal states in the presence of these three types of noise within a common framework have not yet been addressed. The structure of the paper is as follows. Section 2 is devoted to the derivation of the expressions of the contrast with the Bhattacharyya distance and the Fisher ratio in the presence of the three different considered types of noise, and to their comparison and physical interpretation. Two examples of application of these results are given and discussed in Section 3 .

\section{Polarimetric Contrast in the Presence of Different Types of Noise}

We consider active polarimetric imaging systems that illuminate the scene with light whose polarization state is defined by a Stokes vector $\mathbf{S}$ and is produced by a polarization state generator (PSG) (see Fig. 1). We use the Mueller formalism to represent the polarimetric properties of the scene, which is assumed to be composed of two regions: a target characterized by a Mueller matrix $M^{a}$ and a background characterized by a Mueller matrix $M^{b}$. The Stokes vector of the light scattered by the region $a$ (or $b$ ) is given by the equation

$$
\mathbf{S}_{u}=M^{u} \mathbf{S} \text { with } u=\{a, b\} .
$$

The light scattered by the scene is analyzed by a polarization state analyzer (PSA), which is a generalized polarizer whose eigenstate is the Stokes vector T. The final output is a scalar intensity image, in which, at each pixel of region $a$ or $b$, the average signal measured is

$$
i_{u}=\frac{\eta I_{0}}{2} \mathbf{T}^{T} M^{u} \mathbf{S}
$$

where $u=\{a, b\}$ and the superscript $T$ denotes matrix transposition. In this equation, $\mathbf{S}$ and $\mathbf{T}$ are unit intensity, purely polarized Stokes vectors, $I_{0}$ is a number of photons, and $\eta$ is the conversion efficiency between photons and electrons. It is seen in Eq. (2) that, although $i_{u}$ is an intensity measurement, its value depends on the polarimetric properties of the observed regions through their Mueller matrices $M^{u}$ and on the polarization settings of illumination and analysis through vectors $\mathbf{S}$ and $\mathbf{T}$.

We seek to optimize the discrimination between the target of interest and the background. The degrees of freedom available to perform this optimization are the polarization states $\mathbf{S}$ and $\mathbf{T}$. In our case, the discrimination ability depends on the statistical properties of the noise that perturbs the acquisition. However, it is not easy to determine a numerical criterion that quantifies this discrimination and that is comparable for different types of noise statistics. This issue has been addressed, for example, in [12]. It has been shown that, for such real-world discrimination tasks as target detection and object segmentation, the Bhattacharyya distance is a good candidate, although it is only approximate in gener$\mathrm{al}$, and that it is preferable to the Fisher ratio and Kullback-Leibler divergence.

The Bhattacharyya distance is an asymptotic exponent on the probability of error in discrimination problems $[13,14]$. Let us consider two probability density functions (pdfs) $P^{a}(x)$ and $P^{b}(x)$. In our case, these pdfs correspond to the noise statistics of the pixels in regions $a$ and $b$. If $N$ denotes the size of the sample, the probability of error in deciding whether the observed data has been generated with $P^{a}(x)$ and $P^{b}(x)$ behaves as $\exp [-N B]$ as $N$ tends to infinity [14]. Considering our two sets of data defined by their pdfs $P^{a}(x)$ and $P^{b}(x)$, the Bhattacharyya distance is defined as

$$
B=-\ln \left[\int_{\mathcal{D}}\left[P^{a}(x) P^{b}(x)\right]^{1 / 2} \mathrm{~d} x\right],
$$

with $\mathcal{D}$ as the definition domain of $P^{a}$ and $P^{b}$. Please note that the definition in Eq. (3) is valid for continuous random variables defined by probability distribution functions. When dealing with integer-valued

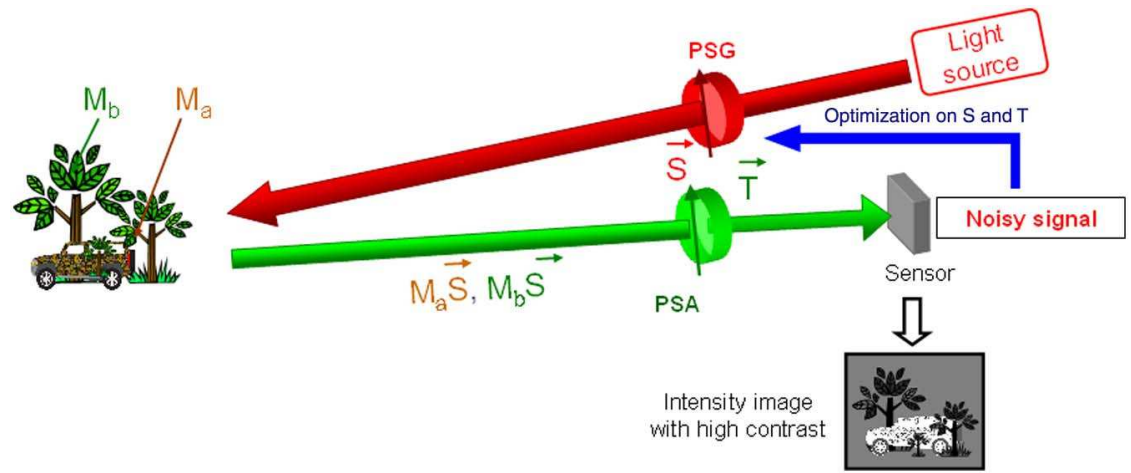

Fig. 1. (Color online) Principle of active scalar polarimetric imaging. PSG, polarization state generator; PSA, polarization state analyzer. 
random variables, such as the Poisson noise addressed in this paper, the integral in Eq. (3) has to be replaced with a discrete sum. The Bhattacharyya distance is thus a scalar value that quantifies the similarity between the pdf $P^{a}(x)$ and $P^{b}(x)$. It belongs to the interval $[0 ;+\infty]$, is equal to zero when the pdfs are identical, and infinite when the pdf supports do not overlap.

Another possible way of quantifying discrimination is the Fisher ratio, expressed as follows:

$$
F=\frac{\left(m_{a}-m_{b}\right)^{2}}{\operatorname{var}_{a}+\operatorname{var}_{b}}
$$

where $m_{u}=\int_{\mathcal{D}} x P^{u}(x) \mathrm{d} x$ and $\operatorname{var}_{u}=\int_{\mathcal{D}} x^{2} P^{u}(x) \mathrm{d} x-$ $m_{u}^{2}$, with $u=\{a, b\}$. The Fisher ratio is equivalent to the Bhattacharyya distance when the noise is Gaussian. For Poisson and speckle noise, it has been shown that the Bhattacharyya distance is more efficient as a measure of discrimination [12]. However, it has to be kept in mind that neither the Bhattacharyya nor the Fisher ratio are ideal scalar measures of discrimination capability. Indeed, except for the case of Gaussian noise, these criteria only approximately satisfy the following desirable property: any scene configuration that leads to the same expression of the measure leads also to exactly the same performance for any optimal signal processing algorithm [15]. However, they have the advantage of being simple to calculate, and thus to optimize, for the considered types of noise. This is why we will use them in the following.

In this section, we will determine the expressions of the Bhattacharyya distance for the three types of noise sources of interest: Gaussian additive noise, Poisson shot noise, and Gamma distributed speckle noise. We will then show that, in the case, important is practice, where the polarimetric properties of the two regions to discriminate are close to each other, the Bhattacharyya distance is well approximated by the Fisher ratio, whose expression makes it easy to compare the three considered noise configurations.

\section{A. Gaussian Additive Noise}

When the intensity backscattered by the source is low enough, the noise from the detector may have a variance larger than that due to the photon shot noise. In this case, the dominant source of noise can be considered additive and Gaussian with a variance independent of the intensity of illumination. The intensity detected by the sensor is then accurately modeled as a Gaussian random variable of mean $i_{u}$ [see Eq. (2)] and with a variance $\sigma^{2}$ independent of $i_{u}$, and the Bhattacharyya distance between the statistical distributions on the target and the background is [12]

$$
B_{\text {gau }}=\frac{\left(i_{a}-i_{b}\right)^{2}}{8 \sigma^{2}} .
$$

In this particular case, the Bhattacharyya distance is proportional to the Fisher ratio, and constitutes a true contrast measure [15].
The distance in Eq. (5) can be expressed in an alternative form that will be useful in the following. Let us define the following intermediate matrices:

$$
M=\frac{M^{a}+M^{b}}{2}, \quad D=\frac{M^{a}-M^{b}}{2} .
$$

By using these two matrices, Eq. (ㅁ) can be written

$$
B_{\text {gau }}(\mathbf{S}, \mathbf{T})=\eta^{2} I_{0}^{2} \frac{\left(\mathbf{T}^{T} D \mathbf{S}\right)^{2}}{8 \sigma^{2}} .
$$

We can see that, in the presence of Gaussian noise, the contrast depends only on the difference $D$ between the Mueller matrices of the target and the background.

In order to optimize imaging, it is interesting to determine the illumination and analysis polarization states $(\mathbf{S}$ and $\mathbf{T})$ that maximize this contrast. In the case of Gaussian noise, Eq. (7) shows that the highest value of $B_{\text {gau }}(\mathbf{S}, \mathbf{T})$ is obtained for

$$
\left(\mathbf{S}_{\text {gau }}, \mathbf{T}_{\text {gau }}\right)=\arg \max _{\mathbf{S}, \mathbf{T}}\left[\left|\mathbf{T}^{T} D \mathbf{S}\right|\right] .
$$

\section{B. Poisson Shot Noise}

When the photon flux backscattered by the scene is large enough, or when the detector noise variance is very low, the photon shot noise arising from the discrete nature of the photons arriving on the detector is the dominant source of noise in the image. In this case, the light coming from the region $u$ can be modeled as a Poisson random variable of mean $i_{u}$ [see Eq. (2)]. In the presence of this type of noise, there does not exist any rigorous definition of the contrast, that is, any single function of the average intensities on the two regions $a$ and $b$ that represents the performance of any signal processing algorithm [12]. However, the Bhattacharyya distance provides a good estimation of the contrast. It takes on the following expression [1]ㄹ:

$$
B_{\mathrm{poi}}=\frac{1}{2}\left(\sqrt{i_{a}}-\sqrt{i_{b}}\right)^{2} .
$$

By using the two matrices $M$ and $D$ defined in Eq. (6), Eq. (9) can be written

$$
B_{\mathrm{poi}}(\mathbf{S}, \mathbf{T})=\frac{\eta I_{0}}{4}\left(\sqrt{\mathbf{T}^{T} M \mathbf{S}+\mathbf{T}^{T} D \mathbf{S}}-\sqrt{\mathbf{T}^{T} M \mathbf{S}-\mathbf{T}^{T} D \mathbf{S}}\right)^{2} .
$$

It is observed that, contrary to the case of additive noise, in the presence of Poisson shot noise, the contrast depends not only of the difference $D$ between the Mueller matrices of the target and the background but also on their sum $M$, that is, on the average Mueller matrix of the whole image. The polarization states that maximize the contrast in the presence of Poisson noise are 


$$
\left(\mathbf{S}_{\mathrm{poi}}, \mathbf{T}_{\mathrm{poi}}\right)=\arg \max _{\mathbf{S}, \mathbf{T}}\left[B_{\mathrm{poi}}(\mathbf{S}, \mathbf{T})\right]
$$

It is important to notice that, in many cases, the Poisson shot noise due to the useful signal, that is, to the light coming from an active source and scattered by the scene, is not the only source of noise. There may be additive noise sources coming from the detector or from the ambient light (for simplicity, we will assume that this latter contribution is unpolarized, so that its intensity does not depend on the PSA setup). Let us assume that the noise source obeys a Poisson statistic and corresponds to a mean number of electrons equal to $i_{n}$. The signals measured from the two regions of the scene are $i_{u}=$ $\eta I_{0} / 2 \times \mathbf{T}^{T} M^{u} \mathbf{S}+i_{n}$ and the expression of the Bhattacharyya distance is obtained by replacing in the above equations the term $\mathbf{T}^{T} \boldsymbol{M S}$ with $\mathbf{T}^{T} \mathbf{M S}+$ $2 i_{n} /\left(\eta I_{0}\right)$.

\section{Gamma Noise}

In the two previous subsections, we assumed that illumination was incoherent. In this case, partially depolarizing Mueller matrices arise from the sum of many incoherent contributions from one resolution cell. If the illumination is coherent, with a coherence length larger than the interaction length inside the materials that constitute the scene, then this sum is coherent within an area called "speckle grain," whose size and shape depends on the characteristics of the imaging system. If the speckle grain is resolved by the sensor, the spatial statistics of the intensity is exponential. If a pixel of the sensor covers $L$ speckle grains, the statistics are Gamma with order $L$ [16]. Thus, in general, the intensity measured by the sensor is Gamma of order $L$ and spatial mean equal to the intensity that would be obtained under incoherent illumination, that is, $\eta I_{0} / 2 \times \mathbf{T}^{T} M^{u} \mathbf{S}$ [17]. In situations where this noise source is dominant with respect to detector and shot noises, the expression of the Bhattacharyya distance is [12]

$$
B_{\text {gam }}=L \ln \left[\frac{1}{2}\left(\sqrt{r}+\frac{1}{\sqrt{r}}\right)\right] \text {, }
$$

where $r=i_{a} / i_{b}$ with $i_{u}$ defined in Eq. (2). If we now introduce the two matrices $M$ and $D$ into Eq. (12), the expression of the Bhattacharyya distance becomes

$$
\begin{aligned}
B_{\text {gam }}(\mathbf{S}, \mathbf{T})= & L \ln \left[\frac { 1 } { 2 } \left(\sqrt{\frac{\mathbf{T}^{T} M \mathbf{S}+\mathbf{T}^{T} D \mathbf{S}}{\mathbf{T}^{T} M \mathbf{S}-\mathbf{T}^{T} D \mathbf{S}}}\right.\right. \\
& \left.\left.+\sqrt{\frac{\mathbf{T}^{T} M \mathbf{S}-\mathbf{T}^{T} D \mathbf{S}}{\mathbf{T}^{T} M \mathbf{S}+\mathbf{T}^{T} D \mathbf{S}}}\right)\right] .
\end{aligned}
$$

This distance can be rewritten as

$$
B_{\text {gam }}(x)=L \ln \left[\frac{1}{2} g(x)\right]
$$

with

$$
g(x)=\sqrt{\frac{1+x}{1-x}}+\sqrt{\frac{1-x}{1+x}}, \quad x=\frac{\mathbf{T}^{T} D \mathbf{S}}{\mathbf{T}^{T} M \mathbf{S}} .
$$

Since the function $g(x)$ is even, it is sufficient to study it as a function of $|x|=\left|\mathbf{T}^{T} D \mathbf{S}\right| / \mathbf{T}^{T} M \mathbf{S}$. We have plotted in Fig. 2 the evolution of $B_{\text {gam }}(x)$ in function of $|x|$. The curve shows that $B_{\text {gam }}(x)$ is an increasing function for $|x| \in[0 ; 1]$ which means that the highest value of the Bhattacharyya distance is obtained when the value of $|x|$ is maximal. Consequently, the states of illumination and analysis that maximize the Bhattacharyya distance verify the following equation:

$$
\left(\mathbf{S}_{\text {gam }}, \mathbf{T}_{\text {gam }}\right)=\arg \max _{\mathbf{S}, \mathbf{T}}\left[\frac{\left|\mathbf{T}^{T} D \mathbf{S}\right|}{\mathbf{T}^{T} M \mathbf{S}}\right] .
$$

It is observed that, in this case again, the contrast depends on both matrices $M$ and $D$.

\section{Comparison of the Contrasts in the Presence of Different Noise Sources}

In order to compare the expressions of the contrast in the presence of the three considered types of noise sources, it is interesting to consider the case where, for all pair of polarization states $(\mathbf{S}, \mathbf{T})$, $\left|\mathbf{T}^{T} M \mathbf{S}\right| \gg\left|\mathbf{T}^{T} D \mathbf{S}\right|$, that is, the difference of the Mueller matrices has a low contribution compared to their sum. That situation is of particular interest since it corresponds to a small difference in the Mueller matrices of the target and background, and, therefore, to the investigation of the detection limit. In this case, we can perform a first-order Taylor expansion of Eqs. (10) and (13) in terms of the ratio $\mathbf{T}^{T} D \mathbf{S} /$ $\mathbf{T}^{T} M \mathbf{S}$, and obtain approximate values of the Bhattacharyya distances for Poisson and Gamma noise sources:

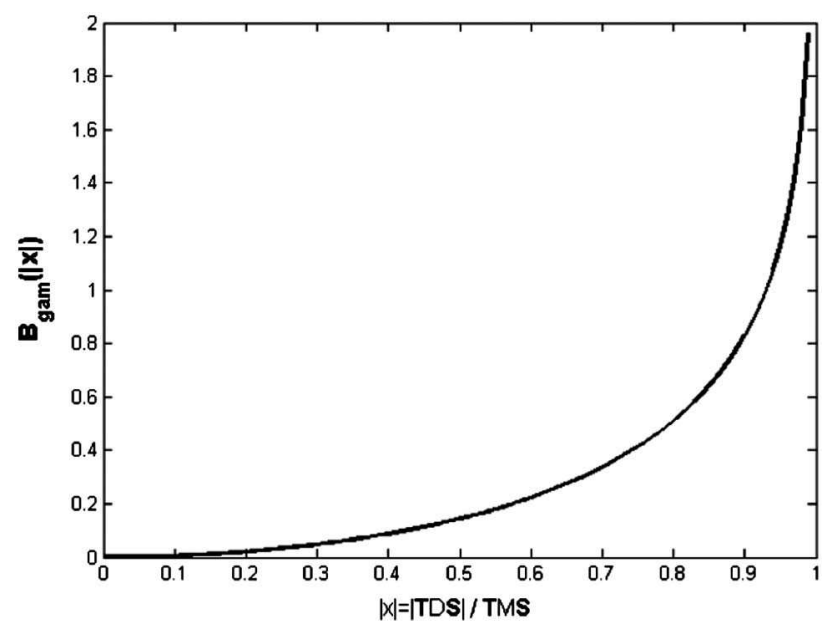

Fig. 2. Evolution of $B_{\text {gam }}(x)$ in function of $|x|=\left|\mathbf{T}^{T} D \mathbf{S}\right| / \mathbf{T}^{T} M \mathbf{S}$ 


$$
B_{\mathrm{poi}}(\mathbf{S}, \mathbf{T}) \simeq \frac{\eta I_{0}}{4} \frac{\left(\mathbf{T}^{T} D \mathbf{S}\right)^{2}}{\mathbf{T}^{T} M \mathbf{S}}=\frac{1}{2} \frac{\left(\eta I_{0} / 2 \times \mathbf{T}^{T} D \mathbf{S}\right)^{2}}{\eta I_{0} / 2 \times \mathbf{T}^{T} M \mathbf{S}}
$$

$$
B_{\text {gam }}(\mathbf{S}, \mathbf{T}) \simeq \frac{L}{2}\left(\frac{\mathbf{T}^{T} D \mathbf{S}}{\mathbf{T}^{T} M \mathbf{S}}\right)^{2}=\frac{1}{2} \frac{\left(\eta I_{0} / 2 \times \mathbf{T}^{T} D \mathbf{S}\right)^{2}}{\left(\eta I_{0} / 2 \times \mathbf{T}^{T} M \mathbf{S}\right)^{2} / L}
$$

These two expressions are, in fact, proportional to Fisher ratios [see Eq. (4)]. Clearly, the term $\eta I_{0} / 2 \times$ $\mathbf{T}^{T} D \mathbf{S}$ present at the numerator of the two expressions represents the difference of mean intensities in the two regions. In the presence of Poisson noise, the denominator is equal to $\eta I_{0} / 2 \times \mathbf{T}^{T} M \mathbf{S}$, which can be seen as the average intensity scattered by the scene and, thus, because of Poisson statistics, as the variance of the fluctuations (which are approximately the same for the two regions since they have similar polarimetric properties). Similarly, the denominator of Eq. (18), $\left[\eta I_{0} / 2 \times \mathbf{T}^{T} M \mathbf{S}\right]^{2} / L$, is the variance of the fluctuations in the scene in the presence of Gamma noise of order $L$.

Comparing these approximate expressions with that of $B_{\text {gau }}$ in Eq. (7) gives insight into the influence of $M$ on the optimal states. In the presence of additive Gaussian noise, the matrix $M$ does not contribute to the value of the Bhattacharyya distance, which depends only on the matrix $D$. In the presence of Poisson and Gamma noise sources, the Bhattacharyya distance does not depend only on $D$ but also on the average intensity through the term $\mathbf{T}^{T} M \mathbf{S}$. It is thus likely that the illumination and analysis states that optimize the contrast will depend on the noise source that affects the image. Namely, in the presence of Poisson and Gamma noise, the optimal states will be the result of a compromise between maximization of the term $\mathbf{T}^{T} D \mathbf{S}$ and minimization of $\mathbf{T}^{T} M \mathbf{S}$.

\section{Application Examples}

We analyze in this section two examples that illustrate the importance of taking into account the noise statistics to determine the optimal illumination and analysis states. The first one is simple and involves only nondepolarizing media. The second one involves depolarizing media and is representative of such applications as remote sensing or imaging through turbid media. In these two examples, the condition $\mathbf{T}^{T} M \mathbf{S} \gg \mathbf{T}^{T} D \mathbf{S}$ is verified, so we will be able to use the Fisher ratio approximations of Eqs. (17) and (18) to interpret the obtained results.

\section{A. Example 1: Linear Diattenuators}

Let us assume that the target and the background behave as linear diattenuators. This is a polarimetric property present in many biological materials [18]. The Mueller matrices of such kind of objects are of the form [19]

$$
P(\gamma, d)=\frac{1}{1+d}\left(\begin{array}{cc}
1 & d \mathbf{v}_{\gamma}^{T} \\
d \mathbf{v}_{\gamma} & \tilde{P}_{\gamma}
\end{array}\right)
$$

In this expression, $\mathbf{v}_{\gamma}=(\cos \gamma, \sin \gamma, 0)^{T}$ is the reduced Stokes vector of the linear eigenstate with azimuth $\gamma$ and transmission $t_{\max }=1$. The orthogonal eigenstate of reduced Stokes vector $-\mathbf{v}_{\gamma}$ has transmission $t_{\min }=(1-d) /(1+d)$, and $d=\left(t_{\max }-t_{\min }\right) /$ $\left(t_{\max }+t_{\min }\right)$ corresponds to the diattenuation. Furthermore, $\tilde{P}_{\gamma}=\alpha I+(1-\alpha) \mathbf{v}_{\gamma} \mathbf{v}_{\gamma}^{T}$, where $I$ is the $3 \times 3$ identity matrix and $\alpha=\left(1-d^{2}\right)^{1 / 2}$. We consider the case where, in a suitably selected coordinates system, $M^{a}=P(\varepsilon / 2, d)$ and $M^{b}=P(-\varepsilon / 2, d)$, which means that the angle between the two diattenuators is equal to $\varepsilon$ and they have the same diattenuation. In this case, it is easily shown that

$$
\begin{aligned}
M & =\frac{1}{1+d}\left(\begin{array}{cccr}
1 & d \cos \varepsilon & 0 & 0 \\
D \cos \varepsilon \alpha+(1-\alpha) \cos ^{2} \varepsilon & 0 & 0 \\
0 & 0 & \alpha+(1-\alpha) \sin ^{2} \varepsilon & 0 \\
0 & 0 & 0 & \alpha
\end{array}\right) \\
D & =\frac{1}{1+d}\left(\begin{array}{cccc}
0 & 0 & d \sin \varepsilon & 0 \\
0 & 0 & (1-\alpha) \cos \varepsilon \sin \varepsilon & 0 \\
d \sin \varepsilon(1-\alpha) \cos \varepsilon \sin \varepsilon & 0 & 0 \\
0 & 0 & 0 & 0
\end{array}\right) .(20)
\end{aligned}
$$

Let us first consider the case of additive Gaussian noise. From Eq. (20), it can be shown that the optimal contrast is obtained when $\mathbf{S}$ and $\mathbf{T}$ are linear and parallel. Let us denote $\theta$ their azimuth. The expression of the Bhattacharyya distance is

$$
\begin{aligned}
B_{\text {gau }}= & \frac{1}{2}\left[\frac{\eta I_{0}}{\sigma(1+d)}\right]^{2}\{\sin \varepsilon \sin 2 \theta(d \\
& +(1-\alpha) \cos \varepsilon \cos 2 \theta)\}^{2}
\end{aligned}
$$

It interesting to analyze the case where $d=1$, and $\varepsilon$ is very small, so that $\cos \varepsilon \simeq 1$ and $\sin \varepsilon \simeq \varepsilon$. In physical terms, that is a situation where the background is fully polarizing, and the target is fully polarizing, as well, but in a direction that makes a small angle $\epsilon$ with the background. In this case, one obtains an expression of $B_{\text {gam }}$ whose maximum is reached for $\theta_{\text {opt }}=30^{\circ}$. This result can be found after cumbersome but elementary calculations. The value of $\theta_{\text {opt }}$ as a function of $\varepsilon$ for different values of $d$ is plotted in Fig. 3. On this graph, it is easy to visualize the evolution of the optimal illumination/ analysis state: when $\varepsilon$ is small, its azimuth is around $30^{\circ}$. As $\varepsilon$ increases, $\theta_{\text {opt }}$ also increases but more slowly, so that when $\varepsilon=90^{\circ}$ (the two diattenuators are orthogonal), $\theta_{\mathrm{opt}}=45^{\circ}$, which means that the optimal state is parallel to one of the diattenuators.

When the image is perturbed with Poisson noise, the states that maximize $B_{\mathrm{poi}}$ are linear and parallel, too. The expression of the contrast is given by Eq. (9) with 


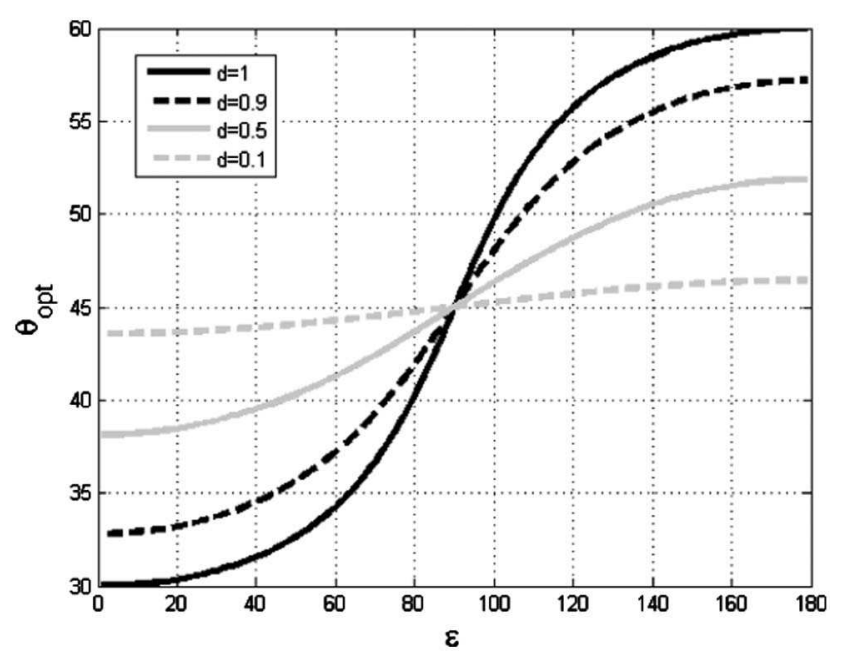

Fig. 3. Variation of $\theta_{\text {opt }}$ as a function of $\varepsilon$ for different values of diattenuation coefficient $d$ in the presence of Gaussian additive noise (Subsection 3.A).

$$
\begin{aligned}
i_{a}= & \frac{\eta I_{0}}{2(1+d)}[(1+\alpha)+2 d \cos (\varepsilon-2 \theta) \\
& \left.+(1-\alpha) \cos ^{2}(\varepsilon-2 \theta)\right], \\
i_{b}= & \frac{\eta I_{0}}{2(1+d)}[(1+\alpha)+2 d \cos (\varepsilon+2 \theta) \\
& \left.+(1-\alpha) \cos ^{2}(\varepsilon+2 \theta)\right] .
\end{aligned}
$$

By deriving this expression, it can be shown that the highest contrast is reached when the relation $\sin (\varepsilon+$ $2 \theta)=-\sin (\varepsilon-2 \theta)$ is verified. The states that maximize $B_{\text {poi }}$ have thus an azimuth $\theta_{\text {opt }}= \pm 45^{\circ}$. Interestingly, this value is independent of the values of $\varepsilon$ and $d$. Thus, in the general case, the optimal states are different in the presence of Poisson and Gaussian noise sources. They are equal only when the two diattenuators are orthogonal $\left(\varepsilon=90^{\circ}\right)$, and the optimal azimuth for Poisson noise is always larger than that for Gaussian noise. This is understandable since, in the case of Poisson noise, one has not only to maximize the term $\mathbf{T}^{T} D \mathbf{S}$ but also to minimize the term $\mathbf{T}^{T} M \mathbf{S}$. This minimization is achieved with azimuth as close as possible to orthogonality with respect to the diattenuators that constitute the scene. Precise computation shows that the optimal compromise is reached for an azimuth of $45^{\circ}$, which is independent of $\varepsilon$.

When the image is perturbed with Gamma noise, it is easy to understand that, to maximize the contrast [Eq. (12)], we have to cancel one of the intensities $i_{a}$ or $i_{b}$, since this will lead to an infinite contrast. This can be done by taking $\mathbf{S}$ and $\mathbf{T}$ orthogonal, one of them being parallel to one of the diattenuators. This configuration allows us to have a region with an intensity almost null and, due to the nature of the Gamma noise, leads to an infinite contrast because there is no noise in this region.

We have represented in Fig. 4(a) the variation of azimuth of the optimal states as a function of the diattenuator angle $\varepsilon$, in the presence of the three sources

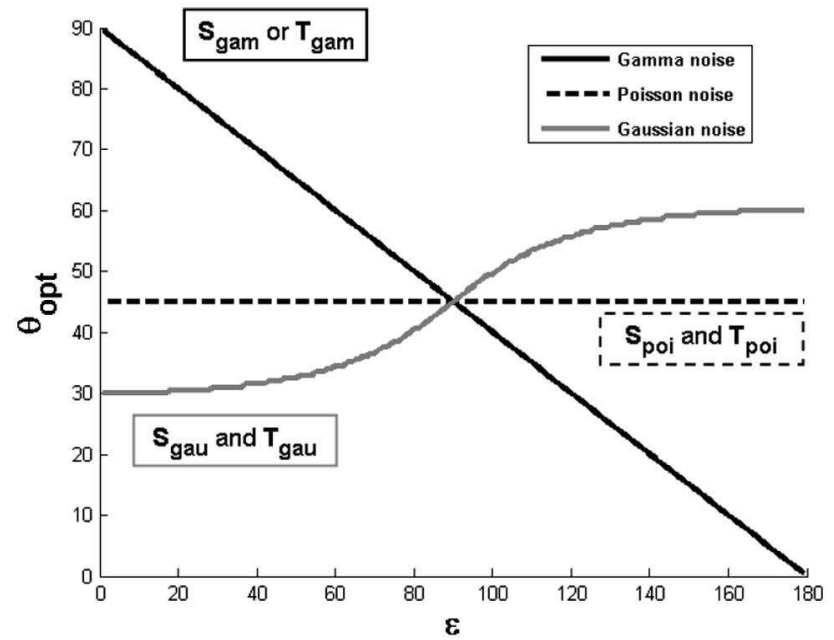

(a)

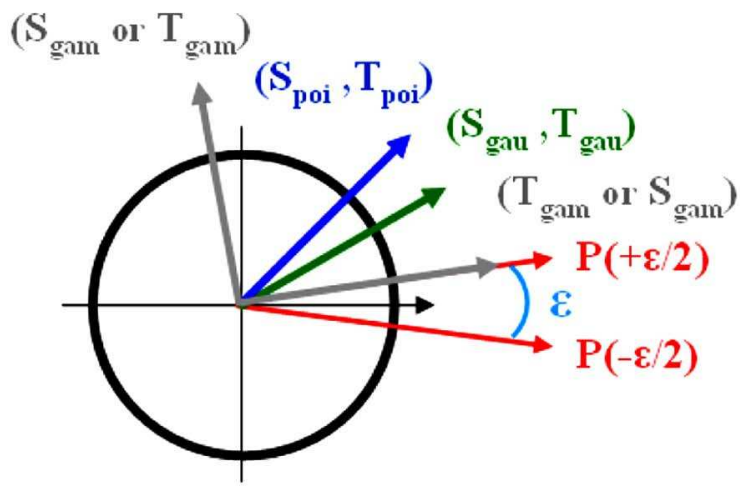

(b)

Fig. 4. (Color online) (a) Variation of $\theta_{\text {opt }}$ as a function of $\varepsilon$ in the scenario of Subsection 3.A, for diattenuation $d=1$, in the presence of Gaussian, Poisson, and Gamma noise sources. (b) Azimuth of the optimal states for the three types of noises, $\varepsilon=20^{\circ}$, and $d=1$.

of noise. Figure $4(\mathrm{~b})$ provide a visual representation of these azimuths when $\varepsilon=20^{\circ}$. Finally, we have represented in Fig. 5 the evolution of the Bhattacharyya distance with $\bar{\varepsilon}$ in the presence of the three considered noise sources. In each part of Fig. 5 , the three curves correspond to the contrasts obtained with the states $\left(\mathbf{S}_{\text {gau }}, \mathbf{T}_{\text {gau }}\right),\left(\mathbf{S}_{\text {poi }}, \mathbf{T}_{\text {poi }}\right)$, and $\left(\mathbf{S}_{\text {gam }}, \mathbf{T}_{\text {gam }}\right)$. As expected, the curve corresponding to the states optimal for the noise source actually present in the image is always above the others. Note that, in the case of Gamma noise [Fig. 4(c)], we have considered that $\mathbf{S}$ and $\mathbf{T}$ are partially polarized $(\|\mathbf{s}\|=\|\mathbf{t}\|=0.99)$ in order to obtain a finite value of the contrast. One also observes that the highest contrast is always reached for the value $\varepsilon=90^{\circ}$ : in this configuration, the two diattenuators are orthogonal and thus easy to distinguish. The optimal states are the same in the presence of the three different noise sources (see Fig. 4): they have an azimuth of $45^{\circ}$.

\section{B. Example 2: Depolarizing Scene}

In the real world, another polarimetric property that is also commonly encountered is the depolarization. Some materials are even characterized only by this 


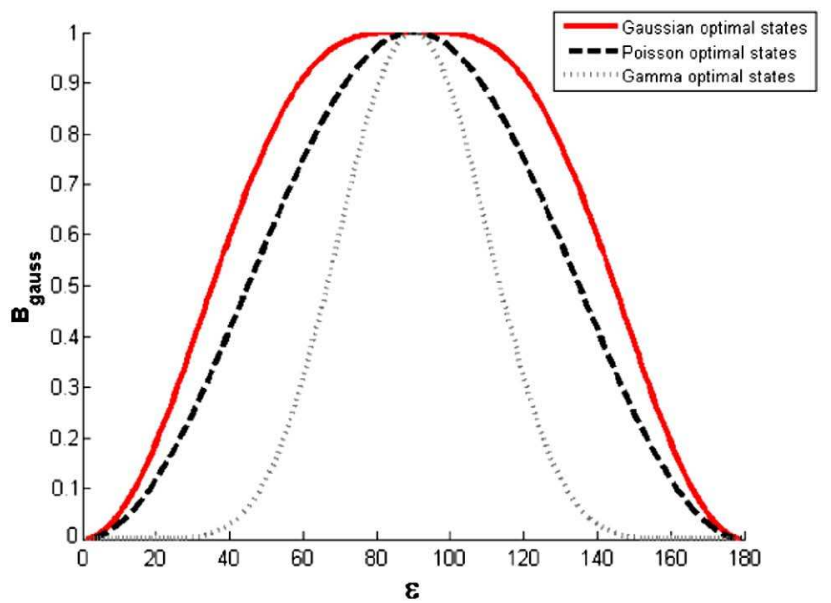

(a)

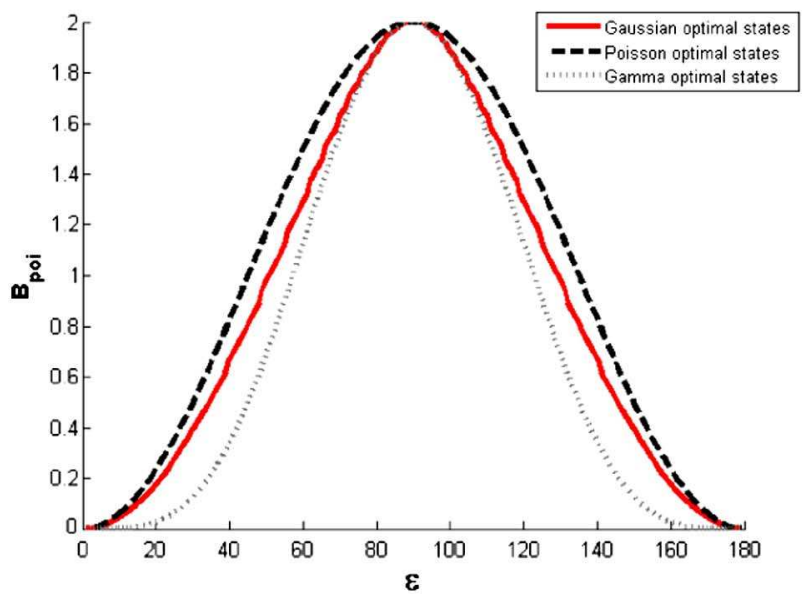

(b)

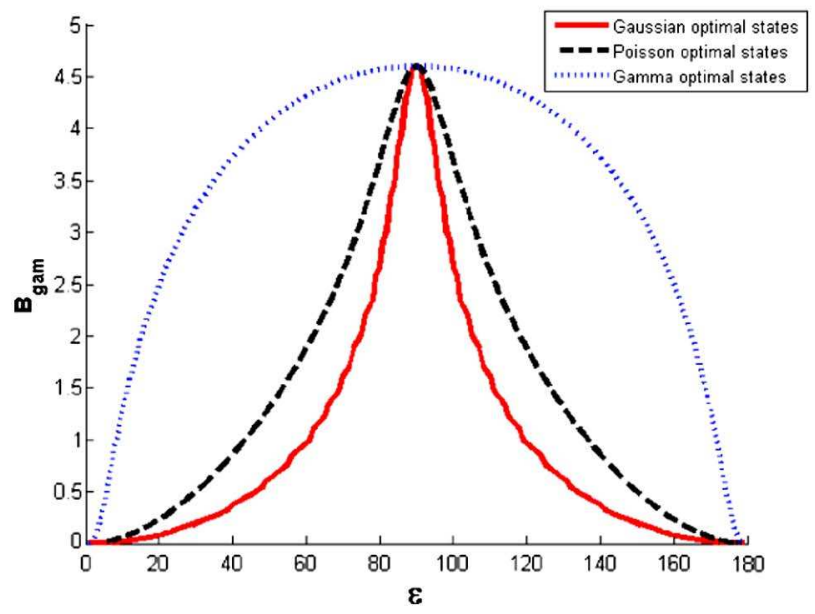

(c)

Fig. 5. (Color online) Evolution of the contrast with parameter $\varepsilon$ in the presence of (a) Gaussian noise, (b) Poisson noise, and (c) Gamma noise, in the scenario of Subsection 3.A, for $d=1$. Figure (c) has been obtained with $\mathbf{S}$ and $\mathbf{T}$ partially polarized $(\|\mathbf{s}\|=\|\mathbf{t}\|=0.99)$ in order to have contrasts that are not infinite.

property [20]. In this example, we consider that the target and the background regions are mainly depolarizing and thus their Mueller matrices are diagonal with the following values:

$$
\begin{aligned}
M^{a} & =\left(\begin{array}{cccc}
0.7 & 0 & 0 & 0 \\
0 & 0.35+\rho & 0 & 0 \\
0 & 0 & 0.35+\rho & 0 \\
0 & 0 & 0 & 0.2
\end{array}\right), \\
M^{b} & =\left(\begin{array}{cccc}
0.8 & 0 & 0 & 0 \\
0 & 0.7 & 0 & 0 \\
0 & 0 & 0.7 & 0 \\
0 & 0 & 0 & 0.45
\end{array}\right),
\end{aligned}
$$

where $\rho$ is a real-valued parameter. They represent media with anisotropic depolarization properties. Such type of Mueller matrices can be obtained when observing biological media that are mainly depolarizing [21]. If we compute the $M$ and $D$ matrices, we have

$$
\begin{aligned}
M & =\left(\begin{array}{cccc}
0.75 & 0 & 0 & 0 \\
0 & 0.525+\rho / 2 & 0 & 0 \\
0 & 0 & 0.525+\rho / 2 & 0 \\
0 & 0 & 0 & 0.325
\end{array}\right), \\
D & =\left(\begin{array}{cccc}
0.05 & 0 & 0 & 0 \\
0 & 0.175-\rho / 2 & 0 & 0 \\
0 & 0 & 0.175-\rho / 2 & 0 \\
0 & 0 & 0 & 0.125
\end{array}\right) .
\end{aligned}
$$

In order to simplify the discussion, we introduce the following notation for the matrices $M$ and $D$ :

$$
M=\left(\begin{array}{cc}
M_{00} & \mathbf{0} \\
\mathbf{0} & \tilde{M}
\end{array}\right), \quad D=\left(\begin{array}{cc}
D_{00} & \mathbf{0} \\
\mathbf{0} & \tilde{D}
\end{array}\right)
$$

where $\tilde{M}$ and $\tilde{D}$ are $3 \times 3$ diagonal matrices. This formalism allows considering the reduced form of the Stokes vectors $\mathbf{S}=\left(1, \mathbf{S}^{T}\right)^{T}$ and $\mathbf{T}=\left(1, \mathbf{t}^{T}\right)^{T}$, where $\mathbf{s}$ and $\mathbf{t}$ are unit norm, three-dimensional vectors.

We represent in Fig. $\underline{6}$ the variation with $\rho$ of the Bhattacharyya distance obtained in the presence of each type of noise. In each of the subgraphs, we have represented the three curves obtained with the optimal states for Gaussian, Poisson, and Gamma noise. Figure 7 shows the evolution of the ellipticity of the optimal states $\mathbf{S}$ and $\mathbf{T}$ with the parameter $\rho$.

Let us first consider that $\rho=0$. By writing Eq. (5) with the parametrization defined in Eq. (25), we obtain $B_{\text {gau }}=\left|D_{00}+\mathbf{t}_{\tilde{D}}^{T} \tilde{D} \mathbf{s}\right|^{2}$. It is seen in Eq. (24) that $D_{00}$ is positive and $\tilde{D}$ is diagonal with maximal coefficient $\tilde{D}_{11}=\tilde{D}_{22}=0.175>0$. In order to reach the highest contrast, the term $\mathbf{t}^{T} \tilde{\tilde{D}} \mathbf{s}$ has to be large and of the same sign as $D_{00}$, which leads to $t$ and $\mathbf{s}$ linear and parallel: the optimal states $\mathbf{S}_{\text {gau }}$ and $\mathbf{T}_{\text {gau }}$ are thus parallel and linear with any value of azimuth since $\tilde{D}_{11}=\tilde{D}_{22}$.

In the presence of Poisson and Gamma noise, the optimal states $\mathbf{S}$ and $\mathbf{T}$ are also linear with any value of the azimuth, since $\tilde{D}_{11}=\tilde{D}_{22}$ and $\tilde{M}_{11}=\tilde{M}_{22}$. 


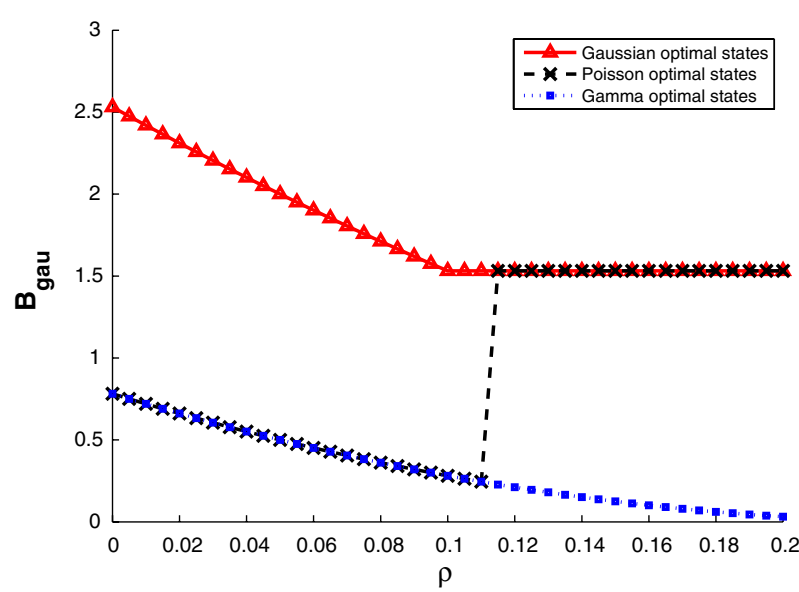

(a)

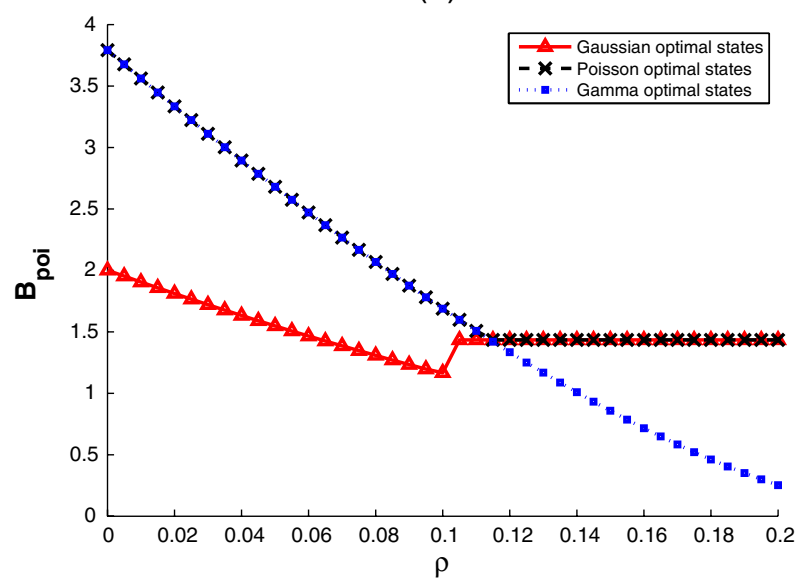

(b)

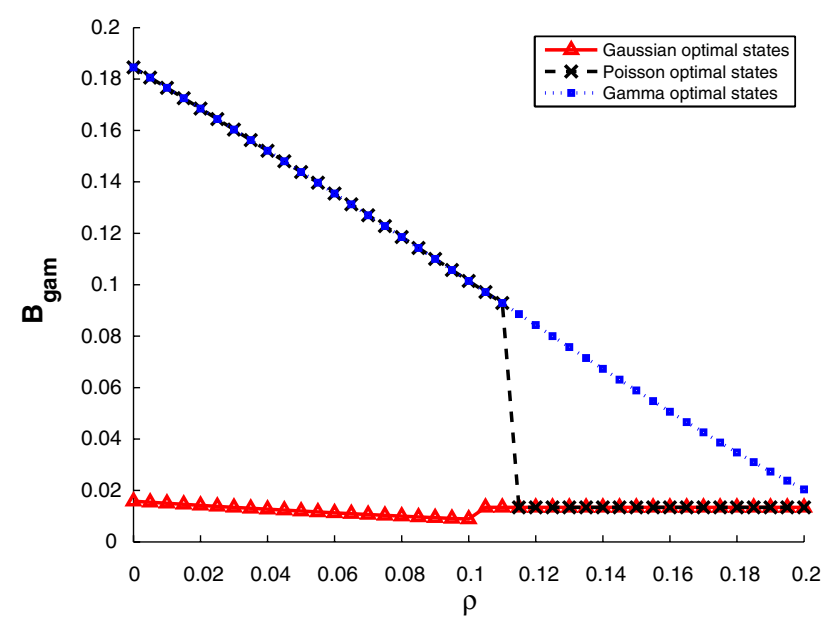

(c)

Fig. 6. (Color online) Evolution of the Bhattacharyya distance with the parameter $\rho$ in the presence of (a) Gaussian noise, (b) Poisson noise, and (c) Gamma noise, for the scenario of Subsection 3.B.

However, they are not parallel but orthogonal. Indeed, we have $\left|\mathbf{T}^{T} M \mathbf{S}\right|=\left|M_{00}+\mathbf{t}^{T} \tilde{M} \mathbf{s}\right|$ and $\left|\mathbf{T}^{T} \tilde{D} \mathbf{S}\right|=$ $\left|D_{00}+\mathbf{t}^{T} \tilde{D} \mathbf{s}\right|$. As $D_{00}$ is small compared to the coefficients of $\tilde{D}$, taking $\mathbf{S}$ and $\mathbf{T}$ parallel or orthogonal (i.e., $\mathbf{t}=\mathbf{s}$ or $\mathbf{t}=-\mathbf{s}$ ) yields almost the same absolute value of $\left|\mathbf{t}^{T} D \mathbf{s}\right|$, but has a larger influence on $\left|\mathbf{t}^{T} M \mathbf{s}\right|$. Since the value of $\mathbf{t}^{T} M \mathbf{s}$ is minimized by taking $\mathbf{S}$ and $\mathbf{T}$ orthogonal to each other $(\mathbf{t}=-\mathbf{s})$, the optimal states in the presence of Poisson and Gamma noise are orthogonal.

As the parameter $\rho$ increases, the coefficients $\tilde{D}_{11}$ and $\tilde{D}_{22}$ decrease and the coefficients $\tilde{M}_{11}$ and $\tilde{M}_{22}$ increase. The coefficients $\tilde{D}_{33}$ and $\tilde{M}_{33}$, associated to the response to circular polarizations, remain constant. In the case of Gaussian noise, as soon as $\tilde{D}_{33}>$ $\tilde{D}_{11}(\rho>0.1)$, the optimal states $\mathbf{S}_{\text {gau }}$ and $\mathbf{T}_{\text {gau }}$ become circular with the same sense of rotation (for the same reason as before). This evolution can be seen in Fig. 7, where the optimal ellipticity goes from 0 to $-45^{\circ}$ for $\rho=0.1$. In Fig. 6 , we can see that, for $\rho \geq 0.1$, the optimal contrast becomes constant. This is because the optimal states $\mathbf{S}_{\text {gau }}$ and $\mathbf{T}_{\text {gau }}$ are circular and the coefficient $\tilde{D}_{33}$ is independent of $\rho$. In the presence of Poisson and Gamma noise sources, for $\rho=0.1$, the optimal states remain linear and orthogonal because, even if the term $\left|\mathbf{T}^{T} D \mathbf{S}\right|$ is smaller for the linear polarization than for the circular one, the term $\left|\mathbf{T}^{T} \mathbf{M} \mathbf{S}\right|$ is also smaller when states are linear.

However, as $\rho$ becomes larger, the terms $\tilde{D}_{11}$ and $\tilde{D}_{22}$ become sufficiently small to overcome the advantage of having larger terms $\tilde{M}_{11}$ and $\tilde{M}_{22}$, and the optimal states $\mathbf{S}_{\text {poi }}$ and $\mathbf{T}_{\text {poi }}$ become circular with the same sense of rotation. It can be seen in Fig. 7 that this happens for $\rho>0.115$, where the ellipticities of $\mathbf{S}_{\text {poi }}$ and $\mathbf{T}_{\text {poi }}$ become equal to $-45^{\circ}$. This change of regime is also observable by a discontinuity of contrast evolution in Fig. 6(b).

In the case of Gamma noise, the optimal states $\mathbf{S}_{\text {gam }}$ and $\mathbf{T}_{\text {gam }}$ remain linear and orthogonal because, even if the term $\left|\mathbf{T}^{T} D \mathbf{S}\right|$ becomes very small, the increase of terms $M_{11}^{a}$ and $M_{22}^{a}$ allows almost annulling the intensity in region $a$. That leads to a fairly low noise in this region and, thus, to a higher Bhattacharyya distance than the one obtained with the circular polarization. The evolution of the ellipticities of the couple $\left(\mathbf{S}_{\text {gam }}, \mathbf{T}_{\text {gam }}\right)$ represented in Fig. 7 confirms this conclusion: they remain the same and equal to zero for any value of $\rho$.

Figure 8 shows images of a target characterized by a Mueller matrix $M^{b}$ and a background characterized by a Mueller matrix $M^{a}$ for $\rho=0.14$, in the presence of Gaussian, Poisson, and Gamma noise sources. For this value of $\rho$, the optimal states associated with the additive Gaussian noise and the Poisson noise are circular and with the same rotation sense, which is why the images of the two first columns are similar. It is seen in this figure that the contrast can be significantly increased if we take the appropriate states of illumination and analysis.

As a summary, we have analyzed in this section the respective influence of the difference between the two Mueller matrices and the average intensities coming from each region of the scene. In the presence of Gaussian noise, only the difference of the Mueller matrices plays a role in the expression of the Bhattacharyya distance. On the other hand, in the 


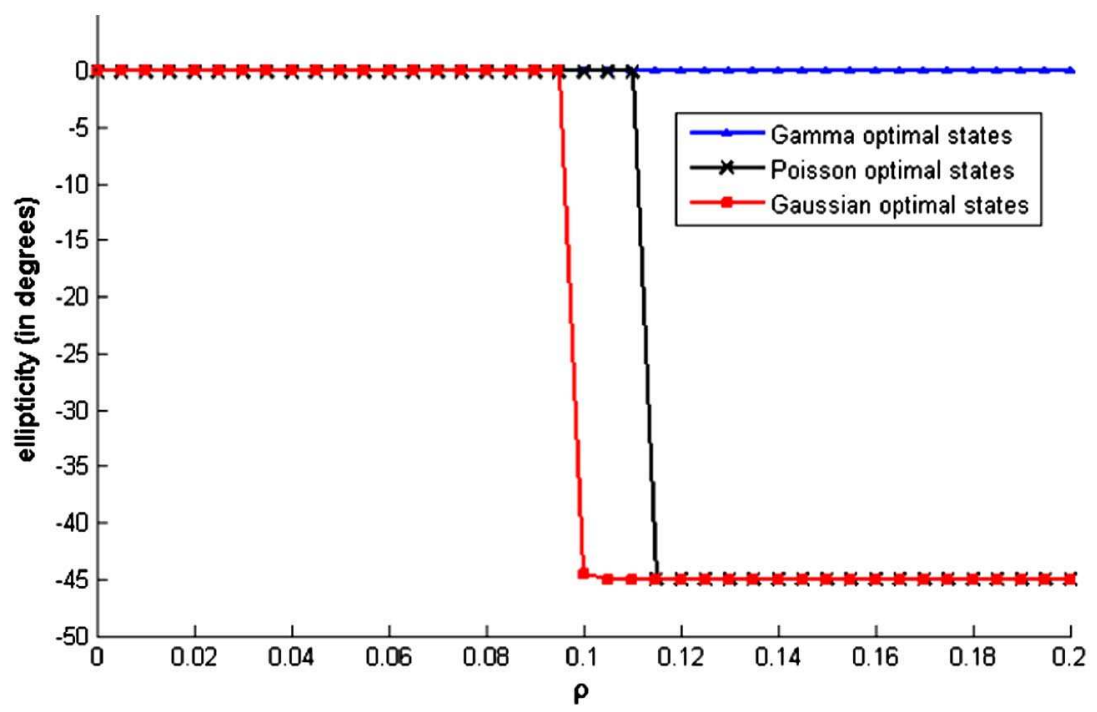

Fig. 7. (Color online) Evolution of the ellipticities of the optimal states $\mathbf{S}$ and $\mathbf{T}$ with the parameter $\rho$, for the scenario of Subsection 3.B.

resence of Gamma noise, it is often preferable to reduce the intensity of one of the regions in order to reduce the noise variance, even if this also reduces the difference between the Mueller matrices. The Poisson noise corresponds to an intermediate configuration. As a consequence, the optimal illumination and analysis states can be different in the presence of Gaussian, Poisson, or Gamma noise, even for the discrimination of simple depolarizing objects. Taking into account the statistics of the noise affecting the image is, thus, of great importance in such applications as imaging in turbid media.

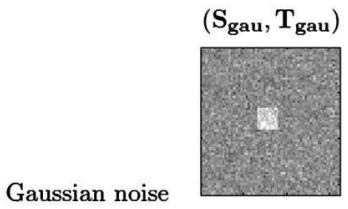

(a) $\mathrm{B}=1.53$

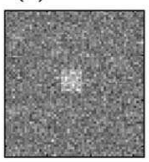

(d) $\mathrm{B}=1.43$

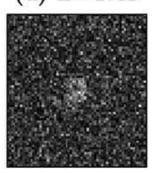

(g) $\mathrm{B}=0.01$

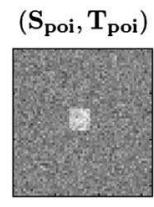

(b) $\mathrm{B}=1.53$

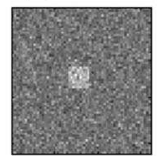

(e) $\mathrm{B}=1.43$

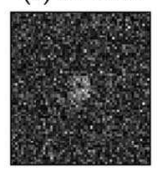

(h) $\mathrm{B}=0.01$

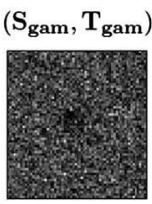

(c) $\mathrm{B}=0.15$
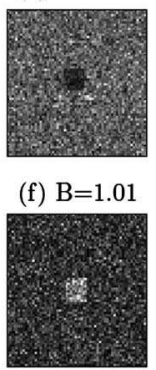

(i) $\mathrm{B}=0.06$
Fig. 8. Images in the presence of (a) - (c) Gaussian, (d)-(f) Poisson, and (h)-(j) Gamma noise of a target characterized by a Mueller matrix $M^{b}$ and a background characterized by a Mueller matrix $M^{a}(\rho=0.14)$, for the scenario of Subsection 3.B. In each case, the image is obtained with optimal states computed with the hypothesis of the presence of Gaussian, Poisson, and Gamma noise (respectively from left to right). The number of photons by pixel is $I_{0}=100$ and the standard deviation of the Gaussian noise is equal to 10 . The Gamma noise is of order $L=1$.

\section{Conclusion}

Considering three types of noise statistics frequently encountered in optical images (Gaussian, Poisson, and Gamma), we have demonstrated that the optimal illumination and analysis polarization states result from a trade-off between the target/background intensity difference and the global intensity in the image. The optimal trade-off between these two effects depends on the noise statistics, and the gain in contrast obtained with the adequate setup is significant. With the advent of versatile and electrically driven polarimetric imagers, the issue of parameter optimization becomes crucial. The results obtained in this paper show that, to perform this task efficiently, the dominant sources of noise have to be carefully identified and taken into account.

This work opens up many perspectives. First, we considered in the present study only noise sources coming from the sensor and from fundamental fluctuations of the light (Poisson and speckle noise). It will be interesting to consider other important sources of noise, such as defects of the polarization modulation components, and consider cases where different types of noise are simultaneously present. We are currently investigating these issues. Second, the study made in this paper relies on the knowledge of the Mueller matrices of the target and of the background. Since they are unknown in practice, some iterative strategies to determine the optimal contrasts have to be investigated.

G. Anna's Ph.D. dissertation is supported by the Délégation Générale pour l'Armement (DGA).

\section{References}

1. J. S. Tyo, M. P Rowe, E. N. Pugh, and N. Engheta, "Target detection in optical scattering media by polarization-difference imaging," Appl. Opt. 35, 1855-1870 (1996).

2. J. E. Solomon, "Polarization imaging," Appl. Opt. 20, 1537-1544 (1981). 
3. R. Walraven, "Polarization imagery," Opt. Eng. 20, 14-18 (1981).

4. A. B. Kostinski and W. M. Boerner, "On the polarimetric contrast optimization," IEEE Trans. Antennas Propag. 35, 988-991 (1987)

5. A. A. Swartz, H. A. Yueh, J. A. Kong, L. M. Novak, and R. T. Shin, "Optimal polarizations for achieving maximal contrast in radar images," J. Geophys. Res. 93, 15252-15260 (1988).

6. J. Yang, Y. Yamaguchi, W.-M. Boerner, and S. Lin, "Numerical methods for solving the optimal problem of contrast enhancement," IEEE Trans. Geosci. Remote Sens. 38, 965-971 (2000).

7. M. Floc'h, G. Le Brun, C. Kieleck, J. Cariou, and J. Lotrian, "Polarimetric considerations to optimize lidar detection of immersed targets," Pure Appl. Opt. 7, 1327-1340 (1998).

8. F. Goudail, "Optimization of the contrast in active Stokes images," Opt. Lett. 34, 121-123 (2009).

9. M. Richert, X. Orlik, and A. De Martino, "Adapted polarization state contrast image," Opt. Express 17, 14199-14210 (2009),

10. F. Goudail, "Comparison of the maximal achievable contrast in scalar, Stokes, and Mueller images," Opt. Lett. 35, 2600-2602 (2010).

11. G. Anna, F. Goudail, and D. Dolfi, "Polarimetric target detection in the presence of spatially fluctuating Mueller matrices," Opt. Lett. 36, 4590-4592 (2011).

12. F. Goudail, P. Réfrégier, and G. Delyon, "Bhattacharyya distance as a contrast parameter for statistical processing of noisy optical images," J. Opt. Soc. Am. A 21, 1231-1240 (2004).
13. T. M. Cover and A. Thomas, Elements of Information Theory Wiley, 1991)

14. A. Jain, P. Moulin, M. I. Miller, and K. Ramchandran, "Information-theoretic bounds on target recognition performance based on degraded image data," IEEE Trans. Pattern Anal. Machine Intell. 24, 1153-1166 (2002).

15. P. Réfrégier and F. Goudail, "Invariant polarimetric contrast parameters for coherent light," J. Opt. Soc. Am. A 19, 1223-1233 (2002).

16. J. W. Goodman, Statistical Optics (Wiley, 1985).

17. K. Kim, L. Mandel, and E. Wolf, "Relationship between Jones and Mueller matrices for random media," J. Opt. Soc. Am. A 4 433-437 (1987).

18. M. Shribak, "Complete polarization state generator with one variable retarder and its application for fast and sensitive measuring of two-dimensional birefringence distribution," $\mathrm{J}$ Opt. Soc. Am. A 28, 410-419 (2011).

19. S. Y. Lu and R. A. Chipman, "Interpretation of Mueller matrices based on polar decomposition," J. Opt. Soc. Am. A 13 1106-1113 (1996).

20. S. Breugnot and P. Clémenceau, "Modeling and performances of a polarization active imager at $\lambda=806 \mathrm{~nm}$," Proc. SPIE 3707, 449-460 (1999).

21. M. Antonelli, A. Pierangelo, T. Novikova, P. Validire, A. Benali, B. Gayet, and A. DeMartino, "Mueller matrix imaging of human colon tissue for cancer diagnostics: how Monte Carlo modeling can help in the interpretation of experimental data," Opt. Express 18, 10200-10208 (2010). 